\title{
Gamification of Educational Environments through Virtual Reality Platforms
}

\section{SIGRADI2018 TECHNOPOLITICAS \\ xxii congresso da sociedade iberoamericana de gráfica digital 22th conference of the iberoamerican society of digital graphics 07|08|09|novembro|2018 iau usp | são carlos | sp br}

\author{
Dr. Juan Pablo Bertuzzi \\ CONICET - FADU-UNL | Argentina | jpbertuzzi@hotmail.com \\ Dr. Arq. Mauro Chiarella \\ CONICET - FADU-UNL | Argentina | mchiarella@hotmail.com
}

\begin{abstract}
This paper proposes the experimentation of new information and communication technologies through the development of an experimental and interactive virtual reality application, where educational content and game mechanics are incorporated, in order to generate interactivity within a digital 3D space and promote academic exchange in an innovative way. This project aims to the exploration of an open 3D digital environment, where the modeling is inspired by some sector of the physical space of the authors' university, compatible for the incorporation of smart objects, avatars and a dialogue/activities system that deals with several educational topics.
\end{abstract}

Keywords: Avatar; Gamification; Hybrid worlds; Techno-politics; Virtual reality.

\section{INTRODUCCIÓN}

A lo largo de la última década, en el marco de las Ciencias de la Información y la Comunicación, los Game Studies y la Interacción Hombre-Máquina, se ha fortalecido el concepto de "mundo híbrido" (Bertuzzi, 2014), a fin de describir el mundo real. Se describe así un mundo constantemente alimentado por dimensiones físicas y digitales, gracias a la evolución de las tecnologías de la información y la comunicación, la interdependencia de los mundos digitales y la proliferación de representaciones digitales, más comúnmente conocidas como avatars. El argumento principal es que este fenómeno, cada vez más presente en la sociedad, es el resultado de un proceso complejo que incluye tres factores específicos e interdependientes.

Para describir el primer factor, es importante comenzar estableciendo la interconexión que existe entre cuatro elementos clave:

- El acto de jugar, entendido como una actividad innata de cada individuo, pero también, siguiendo el trabajo de Roger Caillois (1967), como función social, consustancial con la cultura y con un sentido institucional. Hoy, este elemento se expresa con mayor frecuencia bajo la forma de videojuegos.

- La cultura digital, con una especial atención a las instituciones y a las organizaciones que se dedican al desarrollo y a la experimentación de nuevas tecnologías.

- El acto de la comunicación, visto como un dispositivo in situ y social en las dimensiones digitales y en los mundos persistentes.
- La sociabilidad humana, que evoca constantemente el sentido de los medios de McLuhan (1998), como una extensión de los hombres bajo el crecimiento incesante de las redes sociales.

La combinación de estos cuatro elementos podría visualizarse como un circuito de comunicación donde cada parte se encuentra en constante evolución y en una relación indivisible entre sí. Esta relación se refuerza, en particular, mediante una participación activa de determinados sectores socio-culturales y a través de la implementación de políticas específicas en el desarrollo y en la aplicación de tecnologías experimentales. La idea principal de esta afirmación se sustenta en la representación de una sociedad que se ha acostumbrado a convivir y que también depende de las tecnologías de la comunicación; una sociedad que está constantemente impulsada a consumir una gran cantidad de productos culturales efímeros, en los que los videojuegos ocupan una posición privilegiada como transmisores de mensajes y como productores de discurso.

Por inercia natural, los productos digitales lúdicos se han convertido en una parte importante de la cultura y de las prácticas diarias de muchas comunidades. De hecho, "(...) games are the most successful example of information and communication technologies becoming domesticated, which means that they evidence being integrated into everyday life and practices of groups of people" (Mayra, 2008, p.6). La consiguiente producción y difusión cultural en entornos no físicos, han llevado a experimentar con dimensiones alternativas del desarrollo cognitivo y es así que, por citar un ejemplo, la gamificación de los entornos digitales (considerada aquí como la incorporación de mecánicas de juego en actividades centradas en el aprendizaje), se ha instalado 
como un recurso valioso y como una práctica innovadora en los entornos profesionales y educativos.

El segundo factor es más implícito, ya que depende de un análisis previo de la evolución de los avatars y las interacciones hombre-máquina. Bailenson y Blascovich (2004) afirman que, "within the context of humancomputer interaction, an avatar is a perceptible digital representation whose behaviors reflect those executed, typically in real-time, by a specific human being" (p. 6468). Incluso si el comportamiento de los avatars sigue las órdenes de los seres humanos, estos últimos no siempre están exentos de ser influenciados por sus mismas acciones. La interacción y / o la relación avatar-usuario se ha ido convirtiendo en un elemento clave en la mayoría de los productos digitales, hasta el punto que un gran número de desarrolladores se centran actualmente en la transmisión de emociones desde sistemas complejos de creación de representantes digitales.

Aunque la digitalización del individuo es un proceso que comenzó desde las primeras manifestaciones digitales, el mismo encontró una sólida expresión de valor a partir de la proliferación de redes sociales y de mundos persistentes. Siguiendo el análisis avatar-usuario de Fanny George (2013), la respuesta de los individuos a las proyecciones digitales que los representan varía, pero en numerosos casos estaría relacionada con la construcción de la identidad y de la autoconciencia individual del usuario. En este contexto, la apropiación y la incorporación de representaciones digitales por parte de los usuarios debe tenerse particularmente en cuenta para su posterior análisis.

El último pero no menos importante factor del fenómeno del mundo híbrido incluye la emergencia, la evolución y el desarrollo de las tecnologías de realidad virtual, de realidad mixta y de realidad aumentada, que han hecho concebible el proceso de hibridación incluso de una manera más explícita. Podría ser probable argumentar que estas tecnologías no se encuentran aún instaladas en la mayoría de los sectores sociales y que todavía no pertenecen a las actividades diarias de la mayoría de los individuos. Sin embargo, el uso de estas tecnologías se está naturalizando (y masificando); por lo que se considera que una de las formas más efectivas para conectar definitivamente estos dispositivos en la sociedad, es a través de tecno-políticas y de una mayor participación de espacios educativos y culturales, es decir, mediante la implementación de nuevas tecnologías y representaciones digitales dentro de las infraestructuras educativas.

Las redes sociales, las comunidades digitales y los mundos persistentes son actualmente de gran importancia para el desarrollo social, afectando la productividad de diversos sectores sociales, instituciones, empresas, etc. Además, estas dimensiones digitales mantienen un gran número de sistemas de relaciones sociales a escala global y local. Es posible afirmar que los usuarios están preparados para el uso de tecnologías inmersivas. Por lo tanto, se concluye que la fluidez informacional generada por los sistemas sociotecnológicos es la razón principal para dar dinamismo a la participación funcional del mundo híbrido.

\section{METODOLOGIA}

Este artículo propone la descripción de un proyecto en desarrollo, el cual se encuadra en el marco de la Investigación-Creación y de la Socio-Tecnología, siguiendo una línea de investigación experimental y pluridisciplinaria. Respecto a las Ciencias de la Información y la Comunicación, se mencionan el internet de las cosas, la comunicación social digital, el diseño ético y universal de las NTIC y la teorización de los mundos híbridos anteriormente desarrollada. Respecto del área de especialización de la Interacción HombreMáquina, se pretenden incorporar los conocimientos sobre realidad virtual, sobre la semiótica de las interfaces y sobre un aporte original, basado en el análisis de los "avatars híbridos" (Bertuzzi, 2014), a fin de generar una experiencia múltiple sensorial y física del espacio y la comunicación.

El objetivo general del proyecto es experimentar con nuevas tecnologías de la información y de la comunicación a través del desarrollo de una aplicación experimental e interactiva de realidad virtual, en donde se incorporen contenidos educativos y mecánicas de juego (gamificación), a fin de generar interactividad dentro de un espacio digital 3D y propiciar el intercambio académico de manera innovadora.

Iniciado a fines de noviembre de 2017 bajo la dirección de los autores, este proyecto de investigación y desarrollo de 3 años propone, a su vez, el análisis de las interacciones entre usuarios y avatars, dentro de entornos educativos digitales a través de una plataforma de realidad virtual. Específicamente, se están creando varios escenarios para testear mediante la utilización del sistema de realidad virtual HTC Vive. El desarrollo principal se está construyendo bajo el motor de juego Unity3D (versión 2017.3) y el SDK SteamVR. El objetivo final de este proyecto es generar una dimensión comunicacional innovadora que podría reforzar las hipótesis presentadas a lo largo de este documento.

Pensado para su aplicación inicial en entornos educativos, el análisis y los resultados de esta investigación son dependientes y han sido preparados con respecto a una planificación meticulosa de: la concepción de una plataforma digital 3D; los objetos de juego interactivos; la inteligencia artificial o los avatars manejados por la computadora; la representación humana a través de avatars híbridos; y, por último, el potencial de la inmersión, la ergonomía y la diversidad de control que puede proporcionar el dispositivo de realidad virtual elegido.

Es importante destacar que, a modo de fuente de inspiración, se pueden mencionar las siguientes aplicaciones que han surgido en versión Beta a lo largo de los años 2017 y 2018: "VR Chat" de Unity Technologies; "Pluto VR" de Pluto; y "Ready Room" de Morph3D. Las mismas comparten la idea y logran exitosamente la creación (personal y multiusuario) de avatars y de entornos digitales, con la posibilidad de interactuar con otros usuarios en línea, a través de dispositivos de realidad virtual. Las aplicaciones gratuitas desarrolladas por Google, "Welcome to Light Fields" (exploración de entornos reales a través de realidad virtual) y "Blocks" (herramienta de creación de modelos 
3D en realidad virtual), han sido también de suma importancia a la hora de comprobar la actualidad y el potencial que posee la tecnología en cuestión.

Uno de los desafíos del presente proyecto de investigación es el de poder crear un sistema propio de interacción con avatars y objetos comunicantes, sin depender exclusivamente de las aplicaciones y experiencias mencionadas anteriormente. Esta tarea, si bien más compleja, apunta a resultados a largo plazo, ya que permitirían una mayor libertad a la hora de modificar y/o de crear contenidos originales.

\section{Así, el proyecto presenta dos ejes principales:}

- La primera parte es la estructural, siendo por defecto la piedra angular en vistas de la construcción de un prototipo original. El modelado $3 \mathrm{D}$ en desarrollo ha sido inspirado de un sector específico que pertenece al espacio físico de la universidad de los autores, espacio necesariamente compatible y lo suficientemente flexible para futuras ampliaciones y/o nuevos escenarios. La incorporación de objetos comunicantes, de avatars, de mecánicas de juego, de objetos de juego, más un sistema de diálogo y un sistema de misiones y/o seguimiento de actividades, forman parte del primer prototipo funcional. Estos elementos tienen el objetivo de gamificar el entorno educativo. Como se pretende generar una participación continua y una gran cantidad de interacciones, el mundo digital ha sido compilado para su navegación tanto en un dispositivo convencional (como ser una computadora personal) como en un sistema de realidad virtual (HTC Vive, Oculus Rift y/o ciertos dispositivos para Windows Mixed Reality). Esta decisión se toma, prácticamente, para facilitar la comunicación entre estudiantes y profesores; y estratégicamente, porque ayudará a una población más rápida del entorno digital. Sin embargo, la plataforma digital solo será accesible desde las redes del campus universitario. Las primeras interacciones entre los usuarios y los avatars pertenecientes a la inteligencia artificial, tienen el objetivo inmediato de sensibilizar a los usuarios sobre las posibilidades de las nuevas tecnologías de la información y la comunicación, especialmente en lo que respecta al alcance de los dispositivos de realidad virtual.

- La segunda parte se concentra en la producción de contenidos y en el análisis de datos cualitativos. El desafío es proponer una diversidad de escenarios que obligue a los usuarios a interactuar y a cuestionar ciertos aspectos lúdico-educativos. Por un lado, los usuarios que naveguen en el entorno digital a través de una computadora personal, serán guiados hacia diálogos con personajes no jugadores, a fin de completar misiones en modo individual. El contenido narrativo de estas búsquedas se centra principalmente en los temas tratados en este documento, con un sentido artístico y lúdico. Por otro lado, la experiencia con dispositivos de realidad virtual propone a los usuarios (como es habitual con los dispositivos de realidad virtual) experimentar dentro de una mezcla espacial: es decir, entre un mundo digital aparentemente infinito, pero con una pequeña área física de movimiento. Esta combinación conducirá el contenido narrativo y será crucial para restringir las interacciones del usuario. El punto principal es estimular y generar, en el usuario, una necesidad real de la ayuda de su avatar híbrido (su propia representación digital), para avanzar a través de la experiencia. Al construir una comunicación interna entre la integridad física del usuario y la extensión digital del mismo, las interacciones servirán como auto guía en el mundo digital. Este método es un primer intento, por parte de los autores, de hacer explícitas las temática de la "avatarización", como así también analizar la presencia pasiva del fenómeno de la co-hibridación (conceptos que serán presentados en la última sección del presente artículo).

\section{RESULTADOS}

Se está llevando a cabo el primer prototipo funcional de la plataforma experimental propuesta, la que podrá estar abierta a investigaciones de diversas temáticas. Se pretende así, incorporar futuros proyectos digitales que puedan servirse de la plataforma para experimentar la tecnología de realidad virtual, como también poner énfasis en la producción y en la incorporación de contenidos focalizados en temáticas socio-tecnológicas a venir.

Se han logrado, hasta el momento, los siguientes objetivos de la primera parte del desarrollo:

- Que el usuario aprenda a moverse en el mundo digital de una manera autónoma e intuitiva, a través de una interfaz del usuario intuitiva y de diálogos con avatars manejados por la inteligencia artificial, sin necesidad de explicaciones externas (por ejemplo, sin la necesidad de otra persona que esté a su lado, acompañándolo durante toda la experiencia de realidad virtual).

- Generar interactividad con todos los objetos de juego (GameObjects), los objetos digitales y los objetos comunicantes (avatars) dentro de cada escenario propuesto por la experiencia.

La incorporación de un sistema de navegación/teletransportacion adecuado al modelado 3D de la institución académica elegida.

- Testear la hibridación (físico-digital) a través de la corporeidad (Embodiment) del usuario, mediante la incorporación del cuerpo completo de un avatar (mediante la técnica de cinemática inversa / inverse kinematics), o simplemente a través de la utilización de manos digitales, reemplazando así a los controles renderizados por defecto.

- Incorporar un sistema de diálogos de contenido multinarrativo, sumado a un sistema de seguimiento de misiones y/o actividades.

- Construir dos experiencias que sirven de estructura y/o inspiración para las siguientes etapas de desarrollo: la primera, enfocada a la distribución espacial de objetos digitales; la segunda, que refleja la interacción entre el usuario con avatars manejados por la inteligencia artificial.

Respecto a la integración del sistema de diálogos, el principal desafío fue el de crear una narrativa con múltiples opciones y la posibilidad de la toma de decisiones por parte del usuario. Este punto es clave para
3 
la inmersión del usuario en la experiencia de realidad virtual, ya que permite:

- mantener el interés del usuario con respecto al contenido narrativo, lo que potenciará el aprendizaje sobre temáticas específicas.

- ofrecer la posibilidad de que cada experiencia en el mundo digital sea un poco diferente, lo que podría inducir al usuario a volver a usar la aplicación para probar con otras respuestas y así descubrir información extra.

- mostrar un cierto grado (o al menos la sensación) de inteligencia artificial por parte de los avatars, gracias a la implementación de diferentes reacciones, dependiendo del desenlace o de las respuestas del usuario.

- la posibilidad de entrelazar diferentes contenidos narrativos que se "activen" o que queden ocultos en función del camino tomado por el usuario.

Finalmente, es importante destacar que la posibilidad de tener varias respuestas frente a los diálogos que proponen los avatars es de sumo interés, pero aún más si se incorpora un sistema de actividades y/o de misiones. Esta mecánica de juego permite que los avatars lleven un seguimiento de los diálogos que se van realizando, de las respuestas del usuario y de las actividades o misiones que están en curso, potenciando el nivel de realismo y el sentido narrativo de toda la experiencia.

\section{DISCUSION}

"Quería soñar un hombre: quería soñarlo con integridad minuciosa e imponerlo a la realidad" (Borges, 1993, p. 23). Este pasaje le pertenece al escritor argentino Jorge Luis Borges, que imaginó en su cuento "Las Ruinas Circulares", un hombre taciturno que soñaba sin cesar con la creación de otro hombre. En cada sueño que tenía, estaba un paso más cerca de lograr su cometido a la perfección: estaba soñando a una persona desde la primera molécula hasta cada vena, cada hueso, su carne y su piel, hasta que finalmente llegó a darle una encarnación completa a su creación. Durante las últimas líneas de esta corta historia, el lector puede darse cuenta de que el hombre taciturno también era una ilusión, ya que también era soñado y creado por otra persona. En otras palabras, Borges estaba imaginando un ciclo infinito de proyecciones del yo.

En el mundo híbrido, las personas se proyectan constantemente desde un avatar a otro y, en algunos casos, a través de varios avatars al mismo tiempo. Beth Coleman (2011) argumenta que "the meaning of avatar includes the many modes of representation we employ that make up the different roles we play and places we go" (p. 4). Además, lo que estamos aprendiendo como avatars en el mundo híbrido también está cambiando la percepción del espacio, ya que todo el espectro se está remodelando a través de cada ejemplificación del yo. De esta manera, es importante hacer referencia al concepto de avatar híbrido (línea de investigación de los autores), es decir, una representación digital que tiene el potencial de conglomerar una persona, especialmente en aquellas experiencias diarias que dependen de intercambios constantes entre ambos ambientes físicos y digitales.
Para una mejor comprensión de esta línea de pensamiento, es útil evocar el proceso de coinstanciación de Etienne Amato (2014, p. 52-66), el cual es un proceso que describe una interrelación hombremáquina dentro de una experiencia lúdica, que involucra un modelo comunicacional dinámico, recíproco y en constante evolución. Entonces, una instanciación y coinstanciación continua de individuos está afectando la forma en que los humanos interactúan con los humanos en entornos digitales, ya que éstos también crean instancias de otros usuarios en tiempo real e incluso establecen comportamientos de otros usuarios dependiendo de antiguos y presentes "gameworlds" (Jorgensen, 2014) o mundos digitales. Además, podría ser posible pensar en un arquetipo comunicacional de cohibridación, como una consecuencia automática de todo este fenómeno. La co-hibridación actuaría en este modelo, como la presencia pasiva de muchas instancias de individuos tanto en el mundo físico como en el digital. Esta presencia tendría así una memoria material en donde los avatars híbridos le darían corporeidad.

En este punto, debe quedar claro que la teoría del mundo híbrido es solo un modelo, en el que la progresión de los avatars híbridos es la clave para comprender la naturaleza de todo el sistema de comunicación. De hecho, esta es la razón por la cual las personas piensan colectivamente como individuos, mejorando la multitud y pensando como una multitud de multitudes (en otras palabras, cambiando al lenguaje de programación: como una Clase de Clases de las instancias individuales de cada uno).

Muchas consecuencias pragmáticas podrían ser extraídas de esta conceptualización:

Los avatars híbridos, al ser una mezcla de biología y tecnología, podrían considerarse interacciones digitales internas. A partir de estas interacciones, están surgiendo nuevas formas de inteligencia colectiva, como se muestra en los mundos de juegos en línea y en los universos digitales: formas políticas, mediatizaciones, proyectos informales, todas ellas expresiones que se materializan constantemente en la web. Estas interacciones colectivas también están generando avatars colectivos en un nivel superior, como ser ciudades inteligentes y comunidades inteligentes. Del mismo modo, las organizaciones reales (estructuras sociales, asociaciones, ONGs) se están convirtiendo en avatars informativos, reuniendo una multiplicidad de intencionalidad.

A nivel epistemológico, la incorporación de las herramientas de gamificación en la enseñanza del diseño y la arquitectura que pretende esta investigación, nacen en el marco de las Ciencias de la Información y la Comunicación, los Game Studies y la Interacción Hombre-Máquina, para luego dialogar con las experiencias referentes en el área de las disciplinas proyectuales. El camino ha sido inverso. Tomamos procedimientos, conceptos y metodologías externas a la tradición disciplinar para intentar construir una transversalidad mediada por las nuevas tecnologías.

Como disciplinas reflexivas, la arquitectura y el diseño poseen una dimensión de autonomía en su saber pero su práctica es bastante menos autónoma ya que responde 
con acciones y obras específicas a las demandas provenientes del medio en el cual se ejerce. En sus espacios epistemológicos confluyen los saberes autónomos propios de las disciplinas proyectuales y aquellos que derivan de la heteronomía de sus prácticas sociales. Es así como los clásicos paradigmas científicos positivistas, que han guiado por ejemplo a la formación del arquitecto, la formalización de la profesión y la adopción de los métodos y estrategias del planeamiento territorial en el último siglo, han postergado un debate inconcluso encausando el hacer y pensar del proyecto arquitectónico en occidente dentro de la simple tríada Arte, Ciencia y Tecnología.

El énfasis en la proyectación dentro de la enseñanza de la arquitectura corresponde a la adopción de los métodos y estrategias de la planificación del territorio, la formalización de las incumbencias profesionales y el establecimiento de los mecanismos legales para su ejercicio tanto en el sector público como en el privado. La organización planificada del espacio habitable de las sociedades contemporáneas corresponde al paso de los estados sociales premodernos al mundo de la modernidad. La arquitectura, como una profesión moderna, se rige bajo el peso de los postulados racionalistas y funcionalistas (es decir los mecanismos normativos y de control del espacio habitable) los que usualmente abogan por la construcción de un entorno homogéneo para una sociedad de masas. Bajo estas construcciones epistemológicas, filosóficas e instrumentales (con sus indiscutibles valores y reconocidos defectos) se desarrolla la mayor parte de la enseñanza del proyecto arquitectónico en nuestras comunidades académicas de Latinoamérica.

El uso de la innovación tecnológica tiene un efecto sobre el modo en que los movimientos sociales y las diversas formas de ciudadanía activa operan desde abajo como agentes de innovación, inclusión y desarrollo social. La combinación de las tecnologías innovadoras para alcanzar la participación ciudadana en la toma de decisiones sobre la construcción de la ciudad es una condición esencial para la regeneración ecológica urbana. La discusión sobre la creciente integración de las tecnologías digitales en los espacios urbanos implica una serie de preguntas relacionadas con los complejos procesos de transformación que impactan a las ciudades, como las económicas, sociales, políticas y ambientales. La creación de interfaces y avatars híbridos que posibiliten el desarrollo de pequeñas redes sociales operadas por el acto de la comunicación, es clave como nuevo ambiente de interacción para la enseñanza de las disciplinas proyectuales.

"Humans create meaning by interacting with their natural and social environment, by networking their neural networks with the networks of nature and with social networks. This networking is operated by the act of communication" (Castells, 2012, p. 31). En cuanto a la presente investigación en Ciencias de la Información y la Comunicación, es crucial señalar un análisis más profundo de las Interacciones entre los humanos y los avatars. Como una forma de repensar y reformular el uso humano de los seres humanos (human use of human beings) de Norbert Wiener (1998), es importante saber cuál es el uso de los avatars híbridos por parte de sus usuarios. La respuesta no está tan lejos: los desarrollos actuales y la investigación en realidades virtuales, mixtas y aumentadas demuestran que la forma en que las personas ven el mundo, cambia la forma en que las personas se comunican (comunicación interna y externa) y viceversa. Estas tecnologías ofrecen una forma innovadora de conectar individuos con sus avatars híbridos. Por lo tanto, el diseño digital futuro basado en estas tecnologías también debe considerar una forma potencial de pensar una construcción desde los avatars hacia los humanos.

Para concluir, se insiste en que el presente proyecto en desarrollo, tiene la intención de interrogar métodos alternativos de intercambio institucional, que podrían inspirar la concepción de tecno-políticas innovadoras. A su vez, la propuesta apunta a contribuir especialmente en las áreas de las Ciencias de la Información y la Comunicación; los Game Studies; la Interacción HombreMáquina, como así también al futuro del diseño de experimentaciones con las nuevas tecnologías de realidad virtual.

\section{REFERENCIAS}

Amato, E. (2014). Pour une théorie unificatrice du jeu vidéo: le modèle analytique de la co-instantiation. Psychologie Clinique, 37, 52-66.

Bailenson, J. \& Blascovich, J. (2004). Avatars. In Bainbridge, W. (Ed.), Berkshire Encyclopedia of Human-Computer Interaction (p. 64-68). Great Barrington, MA: Berkshire Publishing Group.

Bertuzzi, J. P., (2014). Mondes Hybrides. Expérimentations sociales pour analyser la relation avatar-utilisateur (Doctoral Thesis, Digital Edition), Paris: Bibliothèque Paris 8.

Borges, J. L., (1993). Las Ruinas Circulares. Ficciones; El Aleph; El Informe de Brodie. Biblioteca Ayacucho.

Caillois, R. (1967). Les jeux et les hommes, Paris: Gallimard.

Castells, M. (2012). Networks of Outrage and Hope: Social Movements in the Internet Age. Malden, MA: Polity Press.

Coleman, B., (2011). Hello Avatar. Rise of the Networked Generation. Cambridge, MA: MIT Press.

Georges, F. (2013). L'avatar comme métaphore conceptuelle et interactive de l'image de soi. In Amato, E. \& Perenny, E. (Eds.), Les avatars jouables des mondes numériques (pp. 135-149). Paris: Lavoisier.

Jorgensen, K. (2014). Gameworld Interfaces. Cambridge, MA: MIT Press.

Mayra, F. (2008). An Introduction to Game Studies, London: SAGE Publications.

McLuhan, M. (1998). La galaxia Gutenberg, Barcelona: Galaxia Gutenberg.

Villagrasa, S., Fonseca, D., Redondo, E. \& Duran, J. (2014). Teaching case of gamification and visual technologies for education. Journal of Cases on Information Technology (JCIT), 16 (4), 38-57.

Wiener, N. (1988). The Human Use of Human Beings: Cybernetics and Society. New York: Da Capo Press. 